ISSN 1997-342X (Online), ISSN 1991-8631 (Print)

Original Paper http://ajol.info/index.php/ijbcs http://indexmedicus.afro.who.int

\title{
Carence en oligoéléments en relation avec la croissance, la production etla composition minérale du haricot en milieu contrôlé
}

\author{
Zéphyrin Nuanisa LUYINDULA \\ Centre National d'Encadrement des Eleveurs et Agriculteurs du Congo (C.N.E.A.C.). \\ 45, avenue Lisala, Commune de Kasa-Vubu, Kinshasa, RDC. \\ E-mail : zeph.luyindula@gmail.com; Tél : +243810024461
}

\section{RESUME}

Le haricot commun est un légume très courant en Afrique, ce qui explique de nombreux travaux de recherche consacrés à cette plante, notamment dans le domaine de sa nutrition minérale. Au cours de cette étude, les carences en principaux microéléments( $\mathrm{Fe}, \mathrm{Zn}, \mathrm{Mn}, \mathrm{Cu}, \mathrm{Mo}, \mathrm{B})$ ont été étudiées chez le haricot dans une serre climatisée. Les plants ont été cultivés en vase de végétation rempli de quart blanc pur comme substrat de culture. La technique utilisée est la méthode de différence, consistant à l'absence d'un des six oligoéléments dans le milieu de culture pour chaque traitement expérimental. Deux témoins, avec absence ou présence total de ces oligoéléments étaient aussi mis en place. Le haricot s'est révélé le plus sensible à l'absence en zinc dans le milieu de culture, qui réduit sensiblement la croissance et la production. Cette absenceen zinc entraîne également un accroissement sensible des teneurs de tous leséléments majeurs et mineurs. La déficience en bore, au contraire, a montré une réduction de teneur en azote, phosphore et potassium chez le haricot, sans affecter la croissance et production.Les effets de la carence des autres éléments mineurs ne se sont que peu manifestés, la croissance, la production et les teneurs en éléments minéraux étant assez proche du 'témoin avec présence de tous les oligoéléments'. Une attention particulière devrait donc être accordée au Zn lors de la culture du haricot, surtout en sol sableux.

(C) 2017 International Formulae Group. All rights reserved.

Mots clés : Haricot commun, microéléments, développement, production,teneurs minérales.

\section{Micronutrient deficiencies as related to the growth, yield and mineral contents of haricot bean in a controlled environmental conditions}

\begin{abstract}
The haricot bean is one of the most common legumes in Africa and a lot of researches are reported to this plant, especially in mineral nutrition aspects. The effects of absence of principal microelements (Fe, $\mathrm{Zn}$, $\mathrm{Mn}, \mathrm{Mo}, \mathrm{B})$ were analyzed on haricot bean in a greenhouse. The haricot beans were planted in the pots with pure quart as culture substrate. The experimental technique used is based on the method of difference; the six micronutrients being absent from the mineral nutrient composition in turn. Two control treatments with or without all the microelements were also added. The results have shown that common bean was very sensible to the absence of $\mathrm{Zn}$ in culture substrate, by highly significant reduction of growth and yield. The $\mathrm{Zn}$ deficiency also increased significantly the macronutrients and micronutrients contents in the haricot bean. However, the B
\end{abstract}


deficiency has reduced N, P and K content of plants, without significantly affected growth and yield. The deficiencies of $\mathrm{Fe}, \mathrm{Mn}, \mathrm{Cu}$ and Mo have no remarkable effects compared with the control treatment with all the micronutrients. One should pay attention on $\mathrm{Zn}$ for haricot bean cultivation,especially planted in sandy soil.

(C) 2017 International Formulae Group. All rights reserved.

Keywords : Common bean, microelements, growth, production, nutrient contents.

\section{INTRODUCTION}

Le haricot commun, Phaseolus vulgaris L., est l'un des légumes à graines la plus cultivée au monde, et plus spécialement en Afrique. En effet, cette plante est une importante source de protéines, vitamines et sels minéraux et joue ainsi un rôle primordial dans la nutrition humaine dans denombreux pays en développement. Dans les zones tropicales et subtropicales, le haricot sec est d'ailleurs appelé « viande des pauvres » car il constitue une alternative d'approvisionnement en protéine et divers éléments minéraux essentiels (Djeugap et al., 2014). Cependant, sa production est considérablement réduite par diverses contraintes biotiques et abiotiques exigeant son amélioration (Ndoutoumou et al., 2015). La nutrition minérale étant un des principales contraintes abiotiques, il se révèle nécessaire de lui consacrer de nombreuses recherches.

Quoique nécessaires en infimes quantités, les oligoéléments, également appelés microéléments, éléments mineurs ou éléments traces, sont aussi indispensables que les macroéléments pour une croissanceet production optimale du haricot. Les principaux microéléments reconnus pour les végétaux sont le zinc $(\mathrm{Zn})$, fer $(\mathrm{Fe})$, bore $(\mathrm{B})$, cuivre $(\mathrm{Cu})$, manganèse $(\mathrm{Mn})$ et molybdène (Mo). Néanmoins, les travaux sur les diverses carences en oligoéléments chez le haricot ne sont ni assez nombreux, ni assez explicites (Muhamba et Nchimbi-Msolla, 2010 ; Nchimbi-Msolla et Muhamba, 2010 ; Ayalew, 2016a,b). Le Zn seraitun des plus importants éléments mineurs et la sensibilité du haricot pour cet élément trace a été mise en évidence par plusieurs auteurs. Selon Hafeez et al. (2013), la déficience en zinc retarde la croissance et affecte également la production $\mathrm{du}$ haricot, le zinc étant essentiel pour l'activation du système enzymatique et son implication dans la photosynthèse (Estevez, 2006 ; Makoi, 2016). Quant au fer, c'est aussi un élément essentiel pour le haricot par son intervention dans la chlorophylle et son rôle majeur dans la photosynthèse. Il intervient aussi dans le transfert d'énergies dans la plante, mais serait également constituant de certains enzymes et protéines (Comeau et al., 2006 ; Estevez, 2006 ; Makoi, 2016). D'autre part, cet élément participe activement dans la nutrition humaine, son manque criant entrainant l'anémie.La carence en fer des plantes se caractérise en général par une forte chlorose des feuilles les plus jeunes, affectant ainsi la croissance des plantes (Estevez, 2006). Quant au cuivreet manganèse, ils ont des fonctions proches en tant que composants essentiels de nombreuses enzymes et interviennent aussi dans le processus de la photosynthèse. Selon Comeau et al. (2006), le manganèse participe à la production d'oxygène moléculaire lors de la photosynthèse, essentiel à l'élongation des racines et à la synthèse des membranes et des substances de défense. Le cuivre joue aussi un rôle spécifique dans la production de vitamine A (Beebe et al., 2000), mais serait également impliqué dans la biosynthèse de la lignine, des alcaloïdes et des phytoalexines, jouant ainsi un rôle contre les maladies (Comeau et al., 
2006; Mokoi, 2016). Malgré leurs besoins très faibles, il a été démontré que le bore et molybdène ont des fonctions très spécifiques chez de nombreuses légumineuses à graines. Le bore agit aussi sur la multiplication cellulaire dans les méristèmes et intervient aussi dans le métabolisme des sucres et leur translocation dans la plante. Le molybdèneactive l'enzyme nitrate réductase qui assure la réduction du nitrate dans les feuilles. (Estevez, 2006). Les symptômes de carence en bore ou molybdène apparaissent d'abord sur les jeunes feuilles.

Cette étude est une contribution dans l'analyseet la connaissance des effets de chacune des carences en oligoéléments essentiels sur le développement et la production du haricot commun, en s'assurant de l'absence totale de ces éléments dans le milieu nutritif. Elle a aussi l'objectif d'expliciterl'incidence de ces déficiences sur la composition minérale de la plante tant en éléments majeurs qu'en microéléments afin de mieux appréhender et expliquer les diverses modifications résultantes de ces carences.

\section{MATERIEL ET METHODES}

\section{Variété et technique de culture}

La variété du haricot utiliséedans cette expérience est dénommée "Prélude ». C'est unevariété naine, à grains blancset donnant des gousses moyennes. Elle est principalement cultivée en Europe, pour l'alimentation humaine comme haricot vert. Cette variété a été choisie non seulement pour sa précocité et sa vigueur, mais aussi étant très courant en milieu paysan et pouvant facilement s'adapter dans des conditions d'Afrique.

La culture s'est faite en vase de végétation en polyvinyle d'une capacité de 10 litres, dans une serre climatisée du laboratoire de Physiologie végétale de l'Université Libre de Bruxelles (Belgique). Le quart blanc concassé de Genève a été utilisé comme substrat de culture placé dans les vases. Avant le semis des graines du haricot, ce quart a été lavé à l'acide chlorhydrique et rincé ensuite plusieurs fois avec de l'eau désionisée afin de le rendre totalement pur, exempt d'éléments minéraux.

Laculture en vase s'est faiteavec percolation récupérée, suivant la méthode décrite par Homès et Van Schoor (1982). Cette méthode consistait à récupérer, pour chaque vase, sa solution minérale, et à le reverser dans la même vase continuellement. Chaque vase avait reçu 4 semences du haricot et, après levée, seulement les deux plants les mieux développés étaient conservés. Cependant, la germination générale a été très bonne et s'est élevée à $96 \%$.

\section{Milieu nutritif et Traitements expérimentaux}

Chaque vase, renfermant 2 plants de haricot, avait bénéficié d'une alimentation minérale de 400 milliéquivalents-grammes $(\mathrm{mEq})$, se répartissant comme suit :

- $\quad$ Anions : azote $(\mathrm{N})=90 \mathrm{mEq}$, soufre $(\mathrm{S})=40$ $\mathrm{mEq}$, phosphore $(\mathrm{P})=70 \mathrm{mEq}$,

- Cations : potassium $(\mathrm{K})=80 \mathrm{mEq}$, calcium $(\mathrm{Ca})=70 \mathrm{mEq}$, magnésium $(\mathrm{Mg})=50 \mathrm{mEq}$.

Les oligoéléments étaient fournis de façon à apporter par vase les quantités totales suivantes :

$\mathrm{Fe}: 320 \mathrm{mg}$ de $\mathrm{FeCl}_{3} \cdot 4 \mathrm{H}_{2} \mathrm{O}$

$\mathrm{Zn}: 40 \mathrm{mg}$ de $\mathrm{ZnCl}_{2}$

B : $120 \mathrm{mg}$ de $\mathrm{H}_{3} \mathrm{BO}_{3}$

$\mathrm{Cu}: 12 \mathrm{mg}$ de $\mathrm{CuCl}_{2} \cdot 2 \mathrm{H}_{2} \mathrm{O}$

$\mathrm{Mn}: 80 \mathrm{mg}$ de $\mathrm{MnCl}_{2} \mathrm{H}_{2} \mathrm{O}$

$\mathrm{Mo}: 0,2 \mathrm{mg}$ de $\left(\mathrm{NH}_{4}\right)_{6} \mathrm{Mo}_{7} \mathrm{O}_{24} \cdot 4 \mathrm{H}_{2} \mathrm{O}$

L'essai expérimental s'est réalisé suivant la méthode de différence. Cette méthode a l'avantage de mieux apprécier la 
contribution de chaque microélément dans la nutrition minérale d'une plante. C'est ainsi que chaque traitement expérimentalétait caractérisé par l'absence d'un des sels minéraux d'oligoéléments dans le milieu nutritif, comme repris dans le Tableau 1. Deux témoins, l'un avec présence et l'autre avec absence totale de tous les oligoéléments, étaient aussi mis en place, ce qui correspondait à un total de 8 traitements expérimentaux pour cette étude.

Le dispositif expérimental était arrangé en blocs randomisés,avec six répétitions. La température de la journée dans la serre a été maintenue à $20^{\circ} \mathrm{C}-25^{\circ} \mathrm{C}$ et l'humidité était de $35 \%$.

\section{Mesures sur la croissance et production, analyses minérales et analyses statistiques}

Les mesures de croissance des haricots se sont réalisées 40 jours après semis et se sont rapportées à la hauteur des plants, et à la longueur et largeur de la première feuille afin de déterminer la surface foliaire. La récolte est intervenue au même moment pour tous les traitements expérimentaux, soit 80 jours après semis, correspondant au stade " haricot vert» pour la variété Prélude.

L'analyse des éléments mobilisés par les plantes s'est fait suivant les techniques d'analyse des végétaux consistant à la méthode de Kjeldahl pour l'azote et spectrométrieà phase gazeuse pour les autres éléments.

Afin de mieux apprécier les effets de carence en oligoéléments, les résultats sont également présentés en pourcentage du témoin PT (témoin avec présence de tous les oligoéléments). L'analyse statistique des données s'est basée sur l'analyse de la variance, suivie du test de comparaison des moyennes (LSD à $5 \%$ et $1 \%$ ), en utilisant le logiciel statistique de Microsoft Excel (Windows 10 Professional, V.1511).

Tableau 1 : Traitements expérimentaux étudiés des divers oligoéléments dans le milieu nutritif des plantes de haricot.

\begin{tabular}{|c|c|c|c|c|c|c|}
\hline \multirow{2}{*}{$\begin{array}{l}\text { Traitements } \\
\text { appliqués }\end{array}$} & \multicolumn{6}{|c|}{ Microéléments dans la solution minérale } \\
\hline & $\mathrm{Fe}$ & $\mathbf{Z n}$ & Mn & $\mathrm{Cu}$ & Mo & B \\
\hline $\mathrm{AFe}$ & - & + & + & + & + & + \\
\hline $\mathrm{AZn}$ & + & - & + & + & + & + \\
\hline AMn & + & + & - & + & + & + \\
\hline $\mathrm{ACu}$ & + & + & + & - & + & + \\
\hline AMo & + & + & + & + & - & + \\
\hline $\mathrm{AB}$ & + & + & + & + & + & - \\
\hline $\mathrm{AT}$ & - & - & - & - & - & - \\
\hline PT & + & + & + & + & + & + \\
\hline
\end{tabular}




\section{RESULTATS}

Symptôme de carence, croissance et production du haricot

Symptômes visibles de carence sur les plantes

La carence en zinc, qui était la plus accentuée, s'est manifestée assez tôt, juste une semaine après la levée. Les plants du haricot du traitement AZn étaient tous rabougris. Les feuilles très petites, déformées, avaient des limbes de couleur jaunâtre, montrant quelques nécroses brun-gris sur les bouts des feuilles. La floraison était peu abondante. Les plants $\mathrm{du}$ traitement témoin avec absence totale d'oligoéléments (AT) ont présenté presque les mêmes symptômes, mais de manière un peu plus prononcée.

La carence en fer du traitement $\mathrm{AFe}$ s'est manifestée principalement sous forme de chlorose ou jaunissement des feuilles. Cette chlorose était localisée sur les feuilles du sommet et du milieu de la plante, les feuilles basales ayant pratiquement conservé la couleur verdâtre. Cette carence a peu affecté la taille et la surface foliaire des plantes (Tableau 2).

La carence en bore est survenue dès la formation des deux premières vraies feuilles. Celles-ci étaient petites, enroulées ou gaufrées, et de couleur vert-foncée. La croissance générale de ces plants étaitsensiblement ralentie durant les deux premières semaines après semis. Cependant, ces symptômes ont ensuite vite disparu et les plantes du traitement $\mathrm{AB}$ ont montré une croissance rapide, aboutissant à un développement presque normal, proche $\mathrm{du}$ traitement PT.

Les plants du haricot des traitements $\mathrm{ACu}$ et $\mathrm{AMn}$, avec absence du cuivre ou manganèse du milieu de culture, ont manifesté des symptômes peu marquées et de façon très tardive. La carence en cuivre s'est présenté sous forme de léger jaunissement des jeunes feuilles, avec parfois présence d'infimes nécroses des pointes et bords supérieurs. Par contre, la carence en manganèse est apparue sur les feuilles sous forme de petits points brunâtres ou grisâtres, à peine visibles.

Aucun symptôme de carence n'a été observé sur les plants du traitement AMo.

\section{Croissance et production du haricot}

Les résultats sur la croissance des haricots, qui s'est rapportée à la hauteur et surface foliaire, et la production sous forme de poidstotal des plantes et des gousses, sont reprisau Tableau 2. Les traitements AZn et $\mathrm{AT}$, qui se sont révélés très proches, se sont caractérisés par une croissance en hauteur et une surface foliaire de loin inférieure à PT et aux autres traitements. En effet, la hauteur et la surface foliaire des traitements AZn et AT n'étaient que de $66 \%$ et $55 \%$ de PT, respectivement. Les autres traitements n'ont montré aucune différence significative entre eux, à l'exception du traitement $\mathrm{AB}$ qui s'est révélé significativement inférieur.

$\mathrm{La}$ production de divers traitements, exprimée en poids secs des gousses et en poids secs total des plants (poids secs des gousses et poids secs de la partie végétative), est aussi présentée dans le Tableau 2. Les traitements AT et AZn ont eu une production en gousses sèches et des poids totaux de loin inférieurs aux autres traitements, ne se situant qu'à environ $30 \%$ de PT. Ces derniers, par contre, n'ont montré aucune différence significative entre eux, à l'exception du traitement $\mathrm{AFe}$ qui s'est révélé significativement inférieur au témoin PT (à 95\% de probabilité uniquement). 


\section{Composition minérale des plantes}

Les teneurs minérales des plantes entières en éléments majeurs et mineurs sont respectivement données au Tableaux 3 et 4.Les traitements expérimentaux, se rapportant à l'absence ou présence des oligoéléments uniquement, ont néanmoins influencé les teneurs en éléments majeurs dans la plante. Le traitement AZn et le témoin sans apport d'oligoéléments (AT) montrent des teneurs en tous les éléments majeurs de loin plus élevées par rapport à PT et aux autres traitements. Le traitement $\mathrm{AFe}$ se caractérise également par des teneurs en N, P et $\mathrm{K}$ assez élevées comparées au PT, tandis que le traitement $\mathrm{AB}$ se caractérise par une tendance contraire au traitement $\mathrm{AFe}$, avec des teneurs faibles pour ces mêmes 3 éléments majeurs.
En ce qui concerne les teneurs en éléments mineurs, l'élément absent dans un traitement se retrouve toujours en plus faible teneur, proche à chaque fois du témoin AT.

Le traitement AZn s'est révélé avec des teneurs les plus élevées en tous les oligoéléments, sauf le Zn qui était l'élément absent du milieu. Cependant, le traitement AT a montré une tendance inverse au traitement AZn. À l'exception des AZn et AT, tous les traitements ont montré des teneurs en $\mathrm{Zn}$ assez élevées, à plus de $9 \%$ par rapport au témoin PT. Il en ait de même pour les teneurs en $\mathrm{Cu}$, en dehors des traitements ACu et AT. Par contre, les teneurs en Fe ont été assez proches pour tous les traitements, même pour le traitement $\mathrm{AFe}(98 \%$ de $\mathrm{PT})$.

Tableau 2 : Croissance des plants du haricot à 40 jours et production en matière sèche par vase $(2$ plants) à la récolte à 80 jours après semis.

\begin{tabular}{|c|c|c|c|c|c|c|c|c|c|c|c|}
\hline Paramètres & Unités & AFe & AZn & AMn & $\mathbf{A C u}$ & AMo & $\mathbf{A B}$ & AT & PT & LSD5\% & LSD1\% \\
\hline \multicolumn{12}{|l|}{ Croissance } \\
\hline \multirow[t]{2}{*}{ Hauteur } & $\mathrm{cm} \% \mathrm{PT}$ & 25,3 & 17,2 & 25,7 & 25,5 & 25,2 & 20,5 & 14,4 & 26,1 & 4,0 & 5,3 \\
\hline & & 97 & 66 & 98 & 98 & 97 & 79 & 55 & 100 & & \\
\hline \multirow[t]{2}{*}{ Surface foliaire } & $\mathrm{cm}^{20} \% \mathrm{PT}$ & 45,1 & 31,2 & 44,9 & 41,1 & 46,7 & 41,8 & 25,8 & 47,6 & 3,1 & 4,2 \\
\hline & & 95 & 66 & 94 & 86 & 98 & 88 & 54 & 100 & & \\
\hline \multicolumn{12}{|l|}{ Production } \\
\hline \multirow[t]{2}{*}{ Poids sec total } & $\mathrm{g} \% \mathrm{PT}$ & 42,1 & 16,6 & 43,8 & 45,1 & 47,3 & 51,8 & 14,9 & 49,3 & 6,9 & 9,3 \\
\hline & & 85 & 34 & 89 & 91 & 96 & 105 & 30 & 100 & & \\
\hline \multirow[t]{2}{*}{ Poids végétatif } & $\mathrm{g} \% \mathrm{PT}$ & 14,6 & 7,8 & 14,7 & 15,0 & 16,2 & 22,0 & 7,3 & 16,5 & 4,5 & 6,2 \\
\hline & & 88 & 47 & 89 & 91 & 98 & 133 & 44 & 100 & & \\
\hline \multirow[t]{2}{*}{ Gousses sèches } & $\mathrm{g} \% \mathrm{PT}$ & 27,5 & 8,8 & 29,1 & 30,1 & 31,1 & 29,8 & 7,6 & 32,8 & 5,2 & 7,1 \\
\hline & & 84 & 27 & 89 & 92 & 95 & 91 & 23 & 100 & & \\
\hline
\end{tabular}


Tableau 3 : Teneurs minérales en macroéléments de la plante entière du haricot de divers traitements expérimentaux, en $\%$ de matière sèche.

\begin{tabular}{|c|c|c|c|c|c|c|c|c|}
\hline & $\mathrm{AFe}$ & $\mathbf{A Z n}$ & AMn & $\mathrm{ACu}$ & AMo & AB & AT & PT \\
\hline $\mathrm{N}$ & 3,55 & 4,32 & 3,27 & 3,29 & 3,22 & 2,75 & 4,11 & 3,10 \\
\hline$\% \mathrm{PT}$ & 115 & 139 & 105 & 106 & 104 & 89 & 133 & 100 \\
\hline $\mathrm{S}$ & 0,13 & 0,16 & 0,13 & 0,11 & 0,14 & 0,16 & 0,14 & 0,13 \\
\hline$\% \mathrm{PT}$ & 100 & 124 & 100 & 87 & 111 & 124 & 111 & 100 \\
\hline $\mathrm{P}$ & 0,54 & 0,81 & 0,51 & 0,50 & 0,49 & 0,42 & 0,70 & 0,47 \\
\hline$\% \mathrm{PT}$ & 115 & 173 & 108 & 106 & 104 & 89 & 148 & 100 \\
\hline $\mathrm{K}$ & 2,82 & 3,28 & 2,70 & 2,62 & 2,50 & 2,05 & 3,44 & 2,42 \\
\hline$\% \mathrm{PT}$ & 116 & 136 & 111 & 108 & 103 & 85 & 142 & 100 \\
\hline $\mathrm{Ca}$ & 0,96 & 1,21 & 0,96 & 0,92 & 0,96 & 1,09 & 1,11 & 1,07 \\
\hline$\% \mathrm{PT}$ & 90 & 113 & 90 & 86 & 90 & 101 & 103 & 100 \\
\hline $\mathrm{Mg}$ & 0,58 & 0,69 & 0,57 & 0,55 & 0,55 & 0,61 & 0,78 & 0,55 \\
\hline$\% \mathrm{PT}$ & 106 & 126 & 104 & 99 & 99 & 110 & 141 & 100 \\
\hline
\end{tabular}

Tableau 4: Teneurs minérales en microéléments de la plante entière du haricot de divers traitements expérimentaux, en ppm de matière sèche.

\begin{tabular}{|c|c|c|c|c|c|c|c|c|}
\hline & AFe & $\mathbf{A Z n}$ & AMn & $\mathrm{ACu}$ & AMo & $\mathbf{A B}$ & $\mathbf{A T}$ & PT \\
\hline $\mathrm{Fe}$ & 59 & 72 & 62 & 62 & 59 & 62 & 60 & 61 \\
\hline$\%$ PT & 98 & 119 & 101 & 102 & 97 & 102 & 99 & 100 \\
\hline Mn & 40 & 42 & 9 & 35 & 38 & 35 & 13 & 38 \\
\hline$\%$ PT & 105 & 109 & 24 & 92 & 99 & 90 & 35 & 100 \\
\hline $\mathrm{Cu}$ & 17 & 18 & 16 & 9 & 15 & 15 & 7 & 14 \\
\hline$\%$ PT & 117 & 127 & 113 & 62 & 104 & 109 & 47 & 100 \\
\hline $\mathrm{Zn}$ & 36 & 24 & 32 & 33 & 32 & 31 & 13 & 28 \\
\hline$\%$ PT & 125 & 85 & 113 & 117 & 112 & 109 & 47 & 100 \\
\hline & $\begin{array}{l}\text { Traiteme } \\
\text { Traitem } \\
\text { Traitem } \\
\text { Traiteme } \\
\text { éments. }\end{array}$ & $\begin{array}{l}\text { absenc } \\
c \text { absen } \\
\text { c absen }\end{array}$ & $\begin{array}{l}\mathrm{r} ; \mathrm{AZn}: \\
\text { hanganès } \\
\text { holybdèn } \\
\text { tous }\end{array}$ & $\begin{array}{l}\text { ment av } \\
\text { u : Trai } \\
\text { : Traite } \\
\text { igoélém }\end{array}$ & $\begin{array}{l}\text { ence de } \\
\text { avec ab } \\
\text { vec abse } \\
\text { PT : Tr }\end{array}$ & bore & & ous les \\
\hline
\end{tabular}




\section{DISCUSSION}

L'analyse de carences en divers éléments mineurs par la méthode de différence apparait efficace pour mieux appréhender les effets de diverses déficiences sur la croissance et la production du haricot. Cependant, seule l'absence en zinc dans le milieu nutritif (AZn) a eu un effet dépressif très marqué. Ce traitement n'a montré aucune différence significative par rapport au traitement témoin avec absence totale d'oligoéléments (AT). Cette étude confirme que le zinc est ainsi le principal oligoélément pour un bon développement de cette plante. Cela a été noté dans plusieurs travaux similaires sur cette plante (Beebe et al., 2000 ; Muhamba et Nchimbi-Msolla, 2010 ; Nchimbi-Msolla et Muhamba, 2010 ; Ayalew, 2016a). En effet, le zinc est un élément primordial par son intervention dans le système enzymatique et la synthèse de chlorophylle et carbohydrate. Il participe ainsi dans la photosynthèse (Estevez, 2006) et synthèse des protéines (Makoi, 2016), ce qui explique la mauvaise croissance et la faible production obtenues en absence de cet élément du milieu de culture du haricot.

L'incidence de l'absence de fer dans le milieu de culture a été faible et peu marquée, bien que quelques symptômes de carence visuelle se fussent manifestés. Néanmoins, aucune différence significative n'a été noté entre les traitements AFe et PT pour tous les paramètres de croissance et production analysés. Pourtant cet oligoélément joue un rôle central dans l'élaboration de la chlorophylle, la photosynthèse et le transfert d'énergie (Comeau et al., 2006; Estevez, 2006 ; Makoi, 2016). Dès lors, comment expliquer la faible incidence de l'absence en fer sur la croissance et la production du haricot cultivé en milieu contrôlé ? Il est fort probable que la graine utilisée, qui renferme des proportions non négligeables en cet élément (50 à 100 ppm) aurait influencée, tant soit-peu, le développement de la plante. En effet, le haricot est très sensible au fer et réagit très positivement même à la faible disponibilité en cet élément (Ayalew, 2016b).

L'effet de l'absence des autres microéléments ( $\mathrm{Mn}, \mathrm{Cu}, \mathrm{B}$ et $\mathrm{Mo})$ sur les paramètres de croissance et production du haricot cultivé en milieu contrôlé a été également faible.Les semences traitées au fongicide et insecticide, mais aussile milieu ambiant, auraient apporté des quantités infimes de ces éléments, cependant assez suffisantes pour un développement quasi normal des plantes.

En ce qui concerne les teneurs en microéléments du haricot en relation avec la nutrition minérale en oligoéléments, l'absence de zinc (AZn) entraîne des teneurs élevées en $\mathrm{Fe}, \mathrm{Mn}$ et $\mathrm{Cu}$ dans la plante, tandis que l'absence en fer (AFe) révèle des teneurs élevées en $\mathrm{Zn}, \mathrm{Mn}$ et $\mathrm{Cu}$. En fait, le rôle physiologique de la plupart des microéléments dans la plante apparaît assez proche, principalement par leurs implications dans le processus de la chlorophylle et la photosynthèse $(\mathrm{Zn}, \mathrm{Fe}, \mathrm{Mn}, \mathrm{Cu})$, ce qui pourrait probablement entraîner le remplacement partiel d'un de ces cations par les autres et permettre une croissance et une production assez normale des haricots.

L'absence d'oligoélément dans le milieu de culture a aussi influencé la composition en éléments majeurs du haricot dans cette étude. Il est à noter que Luyindula et Batanga (2017) ont noté une corrélation significative positive entre $\mathrm{Zn}, \mathrm{Mn}, \mathrm{Cu}$ et les anions $(\mathrm{N}, \mathrm{P})$, mais aussi une corrélation significative négative entre $\mathrm{K}$ et $\mathrm{Zn}$ ou $\mathrm{Mn}$. Cependant, l'accroissement sensible des teneurs en tous les éléments majeurs pour les traitements AZn et AT par rapport aux autres traitements serait principalement dû à l'effet de la concentration, la croissance des plants de ces deux traitements étant très faible. Par contre, les faibles teneurs en $\mathrm{N}, \mathrm{P}$ et $\mathrm{K}$ notées 
pour les plants du traitement $\mathrm{AB}$ pourraient s'expliquer par le retard de croissance de ces plants les premières semaines après levée, suivi ensuite par une élongation rapide des plants, entraînant laréduction des teneurs suite à un effet de dilution des éléments.

Il est également probable que l'absence de divers microéléments du milieu de culture accentue ou attenue une carence suite au phénomène d'antagoniste ou de synergie entre ces oligoéléments. C'est ainsi que l'antagonisme entre le $\mathrm{Fe}$ et $\mathrm{Zn}$, par exemple, a été mise en évidence dans cette étude, le traitement $\mathrm{AFe}$ ayant des teneurs les plus élevées en $\mathrm{Zn}$ et le traitement $\mathrm{AZn}$ des teneurs les plus élevées en Fe.

D'autre part, la méthode expérimentale utilisée, connu comme méthode de différences, en faisant varier les quantités totaleset les proportions d'oligoéléments apportés par traitement, pourrait avoir influencé tant soit-peu les résultats. En effet, la quantité de nutriments apportés pour certains éléments (fer, manganèse par exemple) représentait plus de dix fois la quantité d'autres éléments (zinc, cuivre, molybdène). Des études identiques gardant identiques les quantités totales de microéléments en faisant varier uniquement leurs proportions pourraient s'avérer utiles.

\section{Conclusion}

Les carences en divers oligoéléments ont eu des effets différents sur la croissance, la production et la composition minérale du haricot. Cette plante s'est révélée la plus sensible à la déficience en zinc, qui s'est caractérisée par des symptômes visuels très nets et une réduction sensible de la croissance et production. Alors que la déficience en zinc s'est caractérisée par un accroissement des concentrations en éléments majeurs, la déficience en bore a montré une réduction de teneur en azote, phosphore et potassium chez le haricot. Une attention particulière devrait être accordée à ces deux oligoéléments lors de la culture du haricot, surtout en sols sableux.

\section{CONFLIT D'INTERETS}

Les auteurs déclarent qu'il n'y a aucun conflit d'intérêt en rapport avec cet article.

\section{REMERCIEMENTS}

Mes pensées vont en ce moment aux regrettés Professeurs émérites Homès M.V. et Van Schoor G.H. qui m'ont permis d'approfondir les connaissances sur la nutrition minérale des plantes dans leur laboratoire de Physiologie végétale appliquée des plantes, de l'Université Libre de Buxelles (ULB), en Belgique.

\section{REFERENCES}

Ayalew A. 2016a. Effects of zinc fertilizer on yield and tissue concentrations of manganese, cooper, ironand zinc in leaves and seeds of different haricot bean (Phaseolus vulgaris L.) varieties in SouthernEthiopia. Journal of Resources Development and Management, 17: 6168.

Ayalew A. 2016b. Effects of iron fertilizer on yield and tissue micronutrients concentrations ofdifferent haricot bean (Phaseolus vulgaris L.) varieties in Southern Ethiopia. Food Science and Quality Management, 48: 97-105.

Beebe S, Gonzalez AV, Rengifo J. 2000. Research on trace minerals in the common bean. Food Nutr. Bull., 21: 387-391.

Comeau A, Pageau D, Voldeng H, Brunelle A. 2006. Les oligoéléments. Essentiels à l'établissement du couvert végétal dans les céréales. Grandes Cultures, 16(4): 34-36. 
Djeugap FJ, Mefire MH, Nguefack J, Ngueguim M, Fontem DA. 2014. Effet varietal et dutraitement fongique sur la sévérité de la maladie des taches angulaires et le rendement duharicot commun (Phaseolusvulgaris L.) à l'Ouest-Cameroun. Int. J. Biol. Chem. Sci., 8(3): 1221-1233. DOI: http://dx.doi.org/10.4314/ijbcs.v8i3.33

Estevez B. 2006. L'importance des éléments mineurs : des carences à la toxicité. Une préoccupation en agriculture biologique? Document préparé pour le Syndicat des producteursde grain biologique avec l'appui du Ministère de l'Agriculture, des Pêcheries et de l'alimentation du Quebec, 12 décembre 2006, pp. 1-25

Hafeez B, Khanif YM, Saleem M. 2013. Role of zinc in plant nutrition. A review. Americ. J. Experim. Agric., 3(2): 374391.

Homès MV, Van Schoor G. 1985. Alimentation et fumure minérale des végétaux. Aspects quantitatifs du problème. Acad. Roy. Belg., Mémoires Cl. Sci. (2 ${ }^{\mathrm{e}}$ série), XLIV, pp 1- 360.

Luyindula NZ, Batanga R. 2017. Nutrient contents of young oil palm trees as affected by genetic materials, including some inbred progenies. Intern. J. Res. Agric. Sci.,4(1): 61-65.
Makoi JHJR. 2016. Status of Fe, Cu, Zn and $\mathrm{Mn}$ and their relationship with some physicalchemical properties in the soils of Sonjo and Lupiro alluvial/flood plains in Kilombero and Ulanga Districts, Morogoro, Tanzania. Acad. J. Biotechnol., 4(3): 76-84. DOI: 10.15413/ajb.2015.0225.

Muhamba GT, Nchimbi-Msolla S. 2010. Diversity of common bean (Phaseolus vulgaris L.) genotypes in iron and zinc contents under screen house conditions. African J. Agric. Res., 5(8): 738-747.

Nchimbi-Msolla S, Muhamba GT. 2010. The effects of the environment on iron and zinc concentrations and performance of common bean (Phaseolus vulgaris L.) genotypes. Asian J. Plant Sci., 9(8): 455-462.

Ndoutoumou PN, Toussaint A, Baudouin JP. 2013. Etude histologique des embryons avortéslors des croisements entre Phaseolus vulgaris L. et Phaseolus coccineus L. Int. J. Biol. Chem. Sci., 7(1): 213-224. DOI : http://dx.doi.org/10.4314/ijbcs.v7ili.18 